'Departamento de Medicina General, Integramédica Norte, Santiago de Chile. Médico General.

Trabajo no recibió financiamiento.

El autor declara no tener conflictos de interés.

Recibido el 7 de octubre de 2020, aceptado el 25 de mayo de 2021.

Correspondencia a: Dr. Gustavo Adolfo Ossa V. Santa Inés 1448 , casa 8 , Huechuraba.

Código postal: 3830000 tavo.ossa.vasquez@gmail.com

\section{Síndrome de Sjögren en un adolescente. Caso Clínico}

\author{
GUSTAVO ADOLFO OSSA V. ${ }^{1}$
}

\section{Sjögren syndrome. Report of one case}

Sjögren Syndrome (SS), a slowly progressive disease that has unified, validated diagnostic criteria, with excellent evidence and performance in adults, but not in adolescents. We report a 17 year old teenager with a family history of SS in his sister and mother. He presented with fever and fatigue. He had an elevated $C$ reactive protein, leukopenia, positive antinuclear and anti-Rho antibodies and rheumatoid factor. A scintigraphy showed a severe salivary gland dysfunction. The syndrome in this patient had a pediatric clinical behavior despite his proximity to adulthood.

(Rev Med Chile 2021; 149: 790-795)

Key words: Adolescent; Autoimmunity; Sjogren's Syndrome.
4 1 Síndrome de Sjögren es una enfermedad crónica autoinmune que se caracteriza por la infiltración linfocítica de las glándulas exocrinas, generando xerostomía y xeroftalmia, además de otros síntomas extraglandulares ${ }^{1,2,5}$. Afecta de forma predominante al género femenino (hasta en 90\%) entre la cuarta y sexta década de la vida ${ }^{3,4,7,8}$, con una relación 9:1 mujer-hombre, respectivamente.

Su etiología es multifactorial y para su expresión se requiere la interacción entre varios elementos: predisposición genética (alteración de genes que regulan la síntesis de moléculas pro-inflamatorias), ambientales (infecciones previas por virus Coxsackie, Epstein Barr o citomegalovirus) y hormonales (déficit de estrógenos y receptores estrogénicos) $)^{6,10,13,14}$.

Se clasifica como primario y secundario ${ }^{5,9}$. En el primario no hay otra enfermedad autoinmune asociada, y a su vez se subdivide en enfermedad glandular y extraglandular. El secundario está dado por asociación con otras enfermedades autoinmunes como lupus eritematoso sistémico (LES), artritis reumatoide y esclerosis sistémica9

La prevalencia varía entre 0,01 a 4,8\%; un amplio rango que se debe a diferencias geográficas, grupos de edad y en mayor proporción a diferencias en la aplicación de criterios diagnósticos ${ }^{6,8}$.
En la etapa infantil es una enfermedad rara, con una prevalencia desconocida. La edad promedio del diagnóstico es de nueve años y en la mayoría de estos se ha planteado antecedente de parotiditis crónica recurrente $e^{7,11}$.

Los signos y síntomas más frecuentes son sequedad y disminución de lagrimeo, prurito, sensación de cuerpo extraño, hiperemia de la conjuntiva y fotofobia, xerostomía o sensación de carencia de saliva ${ }^{9,10,13}$. Las manifestaciones extraglandulares están presentes en 30\% y comprometen los sistemas: pulmonar (disnea, tos, dolor torácico, fiebre), hematológico (anemia normocítica normocrómica, leucopenia, volumen de sedimentación globular elevado) e inmunológico (anticuerpos anti-Ro/La, factor reumatoideo y ANA positivos, con gamaglobulinas elevadas $)^{13-15}$.

Para el diagnóstico del SS primario se han propuesto múltiples criterios, dentro de estos, los de mayor exactitud diagnóstica se encuentran: - Criterios de la AECG (American-European Consensus Group).

Presencia de cuatro de los seis criterios que incluyen anticuerpos anti Ro/La o biopsia de glándula salival labial positiva, dan una certeza diagnóstica de 95,2\%, con sensibilidad de $89,5 \%{ }^{9,12,17}$ : 
- Criterios del ACR-EULAR (American College of Rheumatology-European League against Rheumatism) de 2016. Con sensibilidad de $96 \%$ y especificidad de $95 \%{ }^{19}$.

Sin embargo, los anteriores rendimientos en materia de sensibilidad diagnóstica no son extrapolables para niños y adolescentes (solo de $39 \%$ ), debido a que en estos grupos son menos frecuentes las manifestaciones sicca (ojo, boca, piel seca) y aparecen otros síntomas inespecíficos ${ }^{7,9}$. Por ello, desde 1999, Bartunková y cols. proponen los criterios preliminares pediátricos basados en los síntomas, signos y hallazgos de laboratorio más comunes en un grupo de niños entre 10-17 años $^{9,21}$ (Figura 1).

El tratamiento está basado en cuatro pilares:

1. Manejo no farmacológico: aumento de la actividad física.

2. Manejo farmacológico tópico para síntomas sicca locales.

3. Manejo farmacológico para síntomas sicca sistémicos: pilocarpina y cevimelina.

4. Manejo farmacológico para síntomas extraglandulares: corticoides, medicamentos modificadores de enfermedad como hidroxicloroquina, azatioprina, metotrexate, micofenaloto, ciclosporina y medicamentos biológicos como el rituximab, belimumab y abatacept ${ }^{16}$.

\section{Reporte de caso}

Se presenta paciente masculino de 17 años, quien acudió al servicio de consulta ambulatoria del centro médico Integramédica norte por cuadro de 24 h de evolución consistente en dolor esternal punzante, no irradiado, que aumentaba con los movimientos de la caja torácica asociados a fatiga. No asociaba síntomas respiratorios, palpitaciones, fiebre, dolor abdominal ni síntomas urinarios. No tenía antecedentes patológicos personales, ni tóxicos, pero refirió antecedentes familiares reumatológicos (madre y hermana con Sjögren, abuela con LES).

En el examen físico destacó fiebre de $39^{\circ} \mathrm{C}$ con taquicardia de 125 latidos/min, hallazgo de soplo cardiaco sistólico grado II/IV. Se indicó AINE, reposo y se solicitó exámenes de laboratorio. Las pruebas tiroideas, función renal, radiografía de tórax fueron reportados sin alteraciones, el resto de los resultados se reportan a continuación (Figura 2 y Tabla 1 ).

En la consulta de control hubo desaparición del dolor esternal y de la fiebre, sin nuevos síntomas. Ante la PCR elevada de manera importante y su antecedente familiar se sugirió toma de perfil autoinmune, con control de hemograma, PCR y orina, los cuales se muestran en la Tabla 2.

Ante la leucopenia, Anti-Ro, FR y ANA po-

\begin{tabular}{|l|l|}
\hline Síntomas clínicos & $\begin{array}{l}\text { Oral: parotiditis recurrente- Agrandamiento de glándula parótida. } \\
\text { Ocular: queratoconjuntivitis seca, conjuntivitis recurrente. } \\
\text { Mucosas: vaginitis recurrente. } \\
\text { Sistémica: fiebre de origen desconocido, artralgias no inflamatorias, } \\
\text { parálisis hipocaliemica, dolor abdominal. }\end{array}$ \\
\hline Anormalidades inmunológicas & $\begin{array}{l}\text { Presencia de al menos uno de: } \\
\text { - Anti SSA/SSB } \\
\text { - Antinucleares(ANA) } \\
\text { - Factor reumatoide. }\end{array}$ \\
\hline $\begin{array}{l}\text { Otras anormalidades en pruebas de } \\
\text { laboratorio o investigaciones adicionales }\end{array}$ & $\begin{array}{l}\text { - Bioquímica: amilasa sérica elevada } \\
- \text { Hematológica: leucopenia, aumento sedimentación globular. } \\
\text { - Inmunológica: hipergamaglobulinemia policlonal. } \\
- \text { Nefrológico: acidosis tubular renal. }\end{array}$ \\
& $\begin{array}{l}\text { - Prueba histológica de infiltración linfocítica en glándula salival u otros } \\
\text { Órganos. } \\
\text { - Documentación de sequedad ocular: test Shirmer, tinción de bengala. }\end{array}$ \\
\hline Excluir otras enfermedades autoinmunes & - Documentación objetiva de compromiso parotídeo(sialografía). \\
\hline
\end{tabular}

Figura 1. Houghton K., et al. (2005) "Criteria for Juvenile Primary Sjogren's Syndrome". The Journal of Rheumatology $32: 11$. 

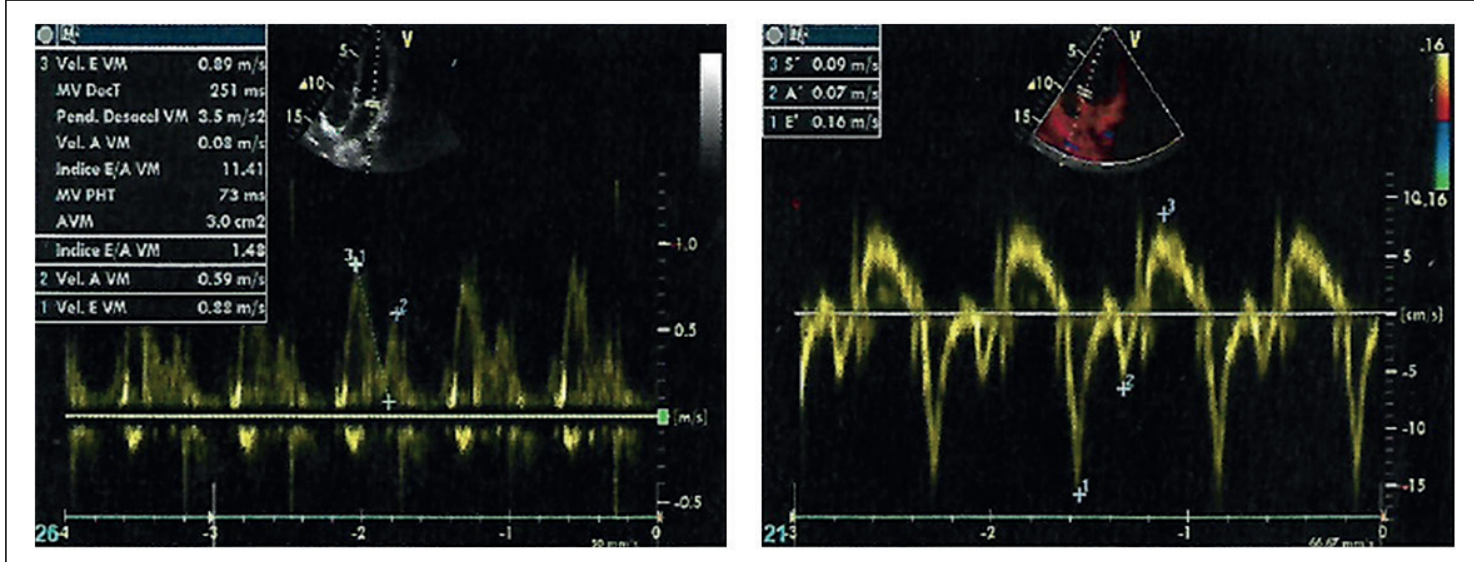

Figura 2. Ecocardiograma transtorácico. Regurgitación tricuspídea mínima.

\section{Tabla 1. Resultado de paraclínicos iniciales}

\begin{tabular}{|lll|}
\hline Paraclínico & Resultado & Valor de referencia \\
Hemograma & Hb: 13,8 & $\mathrm{Hb}: 13,5-17 \mathrm{~g} / \mathrm{dl}$ \\
& Hto: 42,4 & Hto: $40-54 \%$ \\
& GB: 5.260 & GB: $4.000-10.000$ \\
& Plaquetas: 173.000 & Plaquetas: $150.000-400.000$ \\
& VSG: 4 & VSG: $0-13 \mathrm{~mm} / \mathrm{hr}$ \\
Proteína C reactiva & 159 & $0-10 \mathrm{mg} / \mathrm{L}$ \\
Orina completa & Cetonas: $15 \mathrm{mg} / \mathrm{dl}$ & Negativos \\
& Proteínas: $30 \mathrm{mg} / \mathrm{dl}$ & \\
\hline
\end{tabular}

\section{Tabla 2. Resultados de paraclínicos complementarios}

\begin{tabular}{|lll|}
\hline Paraclínico & Resultado & Valores de referencia \\
\hline Anticuerpos antinucleares & $1 / 1.280$ Patrón granular grueso & Negativos \\
\hline Factor reumatoideo & $16,9 \mathrm{UI} / \mathrm{ml}$ & $0-14 \mathrm{UI} / \mathrm{ml}$ \\
\hline Proteína C reactiva & $5 \mathrm{mg} / \mathrm{L}$ & $0-10 \mathrm{mg} / \mathrm{L}$ \\
\hline Hemograma & GB: 3.310 & $4.000-10.000$ \\
\hline Glicemia en ayunas & 83 & $74-99 \mathrm{mg} / \mathrm{dl}$ \\
\hline Orina completa & Proteína indicios & Negativos \\
\hline Ac anti Ro(SS-A) & Cetonas: negativa & \\
Ac anti Ro(SS-A) & Ac Ro52: mayor a $240 \mathrm{U} / \mathrm{ml}$ & Positivos mayor a $10 \mathrm{U} / \mathrm{ml}$ \\
Ac anti La(SS-B) & Ac Ro $60:$ mayor $282 \mathrm{U} / \mathrm{ml}$ & Positivos mayor a $10 \mathrm{U} / \mathrm{ml}$ \\
\hline
\end{tabular}




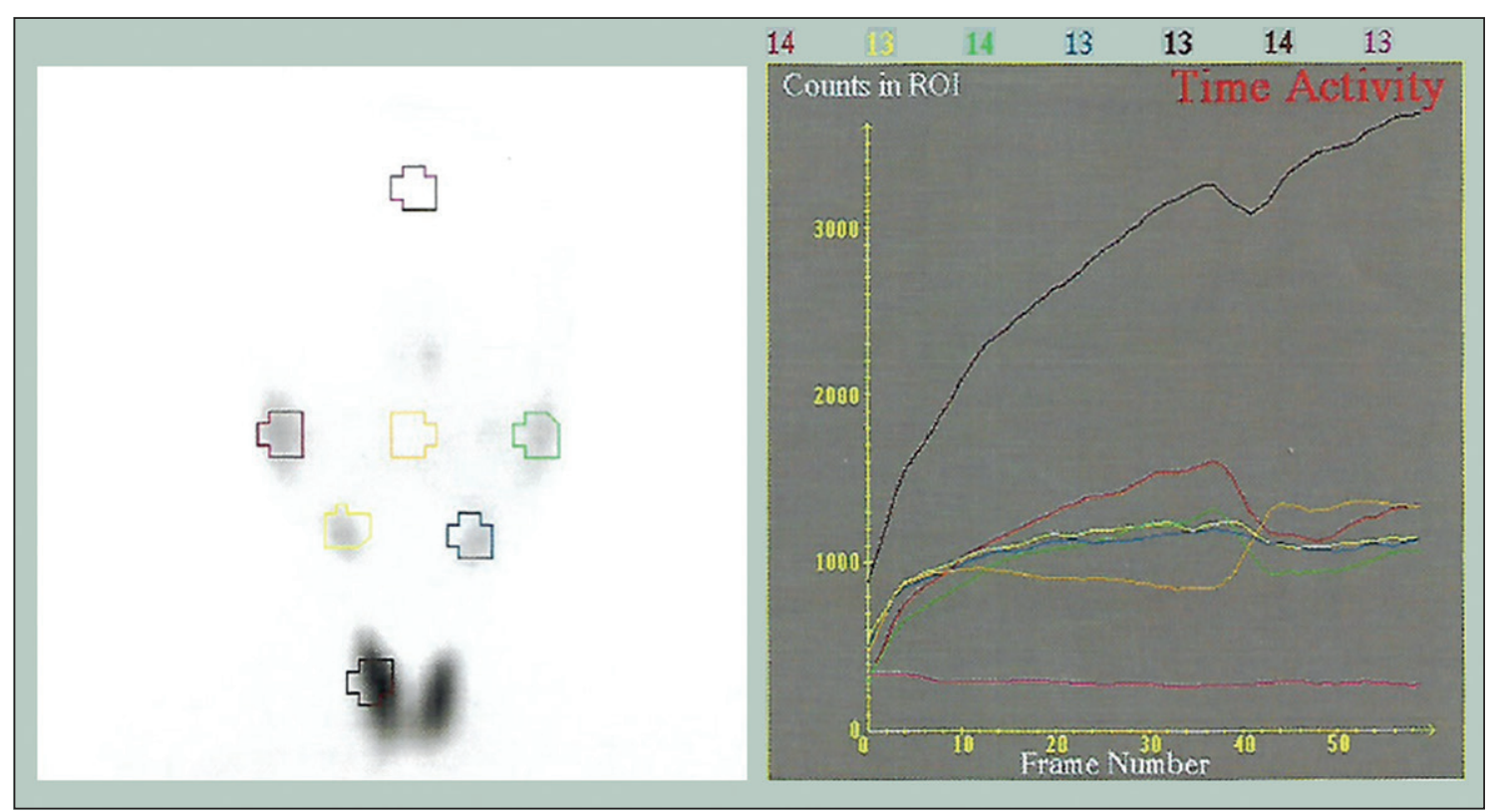

Figura 3. Cintigrama de glándulas salivales. Fracción de eyección global de glándulas salivales de 9\% (valor normal mayor de $30 \%)$.

sitivos es derivado a reumatología y se solicitó cintigrama de glándulas salivales hallándose disfunción franca de las mismas (Figura 3), por lo que inició tratamiento con hidroxicloroquina 200 mg vía oral cada 24 h.

\section{Discusión}

En la práctica clínica, el SS conlleva al médico general a pensar solo en ojo, boca seca y manejo con "lubricantes", además, de ser una enfermedad con poca trascendencia. Sin embargo, es de gran importancia, pues este grupo de pacientes, comparado con la población general, tiene 16 veces más riesgo de desarrollar linfoma, lo que teóricamente afectaría el pronóstico ${ }^{5,10}$. Además, el comportamiento proinflamatorio de la enfermedad ha llevado a plantear el riesgo a largo plazo para el paciente, sobre todo a nivel cardiovascular ${ }^{10,20}$. Por ello, en los últimos quince años, se ha tratado de establecer la asociación entre Síndrome de Sjögren primario y enfermedad ateroesclerótica subclínica, por lo que, en 2018, Chung Yong y cols. realizaron una revisión sistemática y un meta-análisis en los que se observó asociación entre el SS con el aumento de la velocidad de la onda de pulso y grosor de la íntima-media, fenómenos asociados con mayor riesgo de eventos cardiovasculares. Aunque hubo una gran heterogeneidad entre los estudios de este metaanálisis, los resultados alertan al clínico de su implicancia y motivan a los organismos internacionales a realizar más estudios analíticos enfocados en el tema.

En las últimas dos décadas se han analizado reportes de casos aislados de pacientes con SS juvenil encontrándose manifestaciones clínicas y hallazgos de laboratorio muy diferentes en su presentación inicial en comparación con la enfermedad en adultos ${ }^{22}$; por tal motivo, al implementar criterios diagnósticos de adultos en adolescentes, los reportes indican que la sensibilidad es de solo $39 \%$ debido a que en esta población las manifestaciones sicca son menos comunes y pueden no estar presente hasta en $40 \%$ de los casos $^{5,7,9}$. Para darle validez al planteamiento previo, se analizaron los criterios de la ACR-EULAR en el caso del adolescente, encontrando lo siguiente:

- El paciente no tenía síntomas sicca, por lo que para el diagnóstico no se solicitó test de Schirmer, biopsia, ni tinción ocular, perdiendo 5 de 9 puntos. 
- Los anticuerpos anti-La del paciente fueron negativos. En la literatura médica se reportan frecuencias de hallazgos positivos con un promedio de $63 \%$ para los anti-Ro (los cuales fueron positivos y tienen más especificidad en la enfermedad) y menos del $51 \%$ para los anti-La ${ }^{5,9,18}$.

- Flujo salival sin estimular menor o igual a $0,1 \mathrm{ml} / \mathrm{min}$; éste no se llevó a cabo en nuestro paciente, pero de haber sido positivo, apenas sumaría un punto, dejando por fuera el diagnóstico.

No obstante, si al paciente se le aplican los criterios preliminares pediátricos de 1999, se realiza el diagnóstico por cumplir:

- Criterios: clínicos: fiebre, artralgia (articulación esterno-costal). Inmunológicos: Ac anti Ro (SS-A), factor reumatoideo y ANA positivos. Hematológicos: leucopenia. Documentación objetiva de la disminución de la fracción de excreción de las glándulas salivales de 9\%.

Aunque el cintigrama está catalogado como método secundario para diagnóstico, revisiones sistemáticas muestran un rendimiento que oscila entre 47 a $88 \%$ de sensibilidad y 50 a $89 \%$ de especificidad ${ }^{5,18}$, que sumado a sus características (no invasivo, fácil de realizar, bajo costo) lo postulan como una excelente herramienta (no aislada) para llevar a cabo el dictamen. Incluso, en revisiones recientes se ha propuesto la ecografía como herramienta diagnóstica, ya que además de ser accesible y no cruenta, tiene una sensibilidad de hasta $90 \%$ para detectar alteraciones glandulares características de la enfermedad ${ }^{23}$.

Barbara S. et al. presentan en 2011 el caso de tres pacientes (una madre y sus dos hijas), en el cual se pone de manifiesto la necesidad de realizar criterios diagnósticos diferentes por grupo etario y de agregarle mayor importancia al factor genético; esto es congruente con reportes hechos en los últimos 20 años de la asociación familiar ${ }^{24}$, y en este caso, el aspecto de mayor relevancia, porque el antecedente de SS en la madre y hermana fue lo que llevó a sospechar enfermedad reumatológica en el paciente.

\section{Conclusión}

Es necesario validar criterios distintos de los ACR-EULAR para el diagnóstico del Síndrome de Sjögren primario en población pediátrica y adolescente teniendo en cuenta el factor genético; con ello, se tendría un valor más acertado de la prevalencia de la enfermedad y con un diagnóstico temprano, mejoraría el pronóstico en los casos más severos.

\section{Responsabilidades éticas}

Confidencialidad de los datos. El autor declara que ha seguido los protocolos de su centro de trabajo sobre la publicación de datos de pacientes y que el paciente incluido en el reporte (y representante legal) ha recibido información suficiente y ha dado su consentimiento informado por escrito para participar.

\section{Referencias}

1. Al-Hashimi I. The management of Sjögren's Syndrome in dental practice. JADA 2001; 132: 1409-17.

2. Locht H, Pelck R, Manthorpe R. Clinical manifestations correlated to the prevalence of autoantibodies in a large cohort of patients with primary Sjögren's syndrome. A comparison of patients initially diagnosed according to the Copenhagen classification criteria with the American-European consensus criteria. Autoimmun Rev 2005; 4: 276-81.

3. Sutcliffe N. Sjögren's Syndrome. Medicine 2006; 34: 476-9.

4. Nikitakis N, Rivera H, Lariccia C, Papadimitriou J, Sauk J. Primary Sjögren syndrome in childhood: Report of a case and review of the literature. Oral Surg Oral Med Oral Pathol Oral Radiol Endod 2003; 96: 42-7.

5. Takayuki sumida, naoto azuma, masafumi moriyama, hiroyuki takahashi, hiromitsu asashima, fumika honda, et al. clinical practice guideline for sjogren's syndrome 2017. Modern Rheumatol 2018; 28: 383-408.

6. Clio P. Mavragani, Haralampos M. Moutsopoulos. The geoepidemiology of Sjögren's syndrome. Autoimmunity Reviews 2010; 9: A305-10.

7. Gabriel SE, Michaud K. Epidemiological studies in incidence, prevalence, mortality, and comorbidity of the rheumatic diseases. Arthritis Res Ther 2009; 11: 229.

8. Haugen AJ, Peen E, Hultén B, Johannessen AC, Brun JG, Halse AK, et al. Estimation of the prevalence of primary Sjögren's syndrome in two age-different communitybased populations using two sets of classification criteria: the Hordaland Health Study. Scand J Rheumatol 2008; 37: 30-4. 
9. Bartůnková, A Sedivá, J Vencovský, V Tesar. Primary Sjögren's syndrome in children and adolescents: proposal for diagnostic criteria. Clinical and Experimental Rheumatology 1999; 17 (3): 381-6.

10. Cafaro G, Croia C, Argyropoulou OD, Leone MC, Orlandi M, Finamore F, et al. One year in review 2019: Sjögren's syndrome. Clinical and Experimental Rheumatology 2019.

11. Baszis K, Toib D, Cooper M, French A, White A. Recurrent parotiditis as a presentation of primary pediatric Sjögren syndrome. Pediatrics 2012; 129 (1): e179-82.

12. Vitali C, Bombardieri S, Jonsson R, Moutsopoulos HM, Alexander EL, Carsons SE, et al. Classification criteria for Sjögren's sindrome: a revised version of the European criteria proposed by the American-European Consensus Group. Ann Rheum Dis 2002; 61: 554-8.

13. Diez Morrondo C, Lema Gontad JM, Alvarez Rivas N, Atanes Sandoval A, De Toro Santos FJ, Pinto Tasende JA, et al. Aspectos actuales del sindrome de Sjogren: etiopatogenia, manifestaciones clinicas, diagnóstico y tratamiento. Seminarios de la Fundación Española de Reumatología. 2010; 11 (2): 70-6.

14. Karaiskos D, Mavragani CP, Makaroni S, Zinzaras E, Voulgarelis M, Rabavilas A, et al. Stress, coping strategies and social support in patients with primary Sjogren's syndrome prior to disease onset: a retrospective case-control study. Ann Rheum Dis 2009; 68 (1): 40-6.

15. Gono T, Kawaguchi Y, Katsumata Y, Takagi K, Tochimoto A, Baba S, et al. Clinical manifestations of neurological involvement in primary Sjogren syndrome. Clin Rheumatol. 2011; 30: 485-90.

16. Price EJ, Rauz S, Tappuni AR, Sutcliffe N, Hackett KL, Barone F, et al. The British Society for Rheumatology guideline for the management of adults with primary Sjogren's Syndrome. Rheumatology 2017;56: e24-e48.
17. Houghton K, Malleson P, Cabral D, Petty R, Tucker L. Primary Sjögren's syndrome in children and adolescents: are proposed diagnostic criteria applicable? J Rheumatol. 2005; 32: 2225-32.

18. Trevisani VFM, Pasoto SG, Fernandes MLMS, Lopes MLL, de Magalhães Souza Fialho SC, Pinheiro AC, et al. Recommendations from the Brazilian society of rheumatology for the diagnosis of Sjögren's syndrome (Part I): glandular manifestations (systematic review). Adv Rheumatol 2019; 59: 58.

19. Shiboski CH, Shiboski SC, Seror R, Criswell LA, Labetoulle M, Lietman TM, et al. The International Sjogren's Syndrome Criteria Working Group American College of Rheumatology/European League Against Rheumatism Classification Criteria for Primary Sjogren's Syndrome. Arthritis Rheumatol. 2017; 69 (1): 35-45.

20. Yong WC, Sanguankeo A, Upala S. Association between primary Sjogren's syndrome, arterial stiffness, and subclinical atherosclerosis: a systematic review and meta-analysis. Clin Rheumatol. 2019; 38 (2): 447-55.

21. Houghton K, Malleson P, Cabral D, Petty R, Tucker L. Primary Sjögren's syndrome in children and adolescents: are proposed diagnostic criteria applicable? J Rheumatol. 2005; 32 (11): 2225-32.

22. de Souza TR, Silva IH, Carvalho AT, Gomes VB, Duarte AP, Leão JC, et al. Juvenile Sjögren syndrome: distinctive age, unique findings. Pediatr Dent 2012; 34: 427-30.

23. Nieto González JC, Monteagudo Sáez I, Serrano Benavente B. Síndrome de Sjögren. Protoc Diagn Ter Pediatr 2020; 2: 187-94.

24. Longhi BS, Appenzeller S, Centeville M, Gusmão RJ, Marini R. Primary Sjögren's syndrome in children: is a family approach indicated? Clinics (Sao Paulo) 2011; 66 (11): 1991-3. 